\title{
Affective Interaction: Challenges at the Ubiquitous Computing Times
}

\author{
Stephane Gros, Jerome Dupire, and Stephane Natkin \\ CNAM - CEDRIC, 292 rue St Martin 75003 Paris, France \\ stephane.gros@gmail.com, \{dupire, natkin\}@cnam.fr
}

\begin{abstract}
Due to the diversity of studies towards detection of affective state of a user, it is hard to distinguish amongst them toward specific needs. Our approach consists in realizing a comparative study taking in account specificities such as mobility to be able to choose the best settings for a specific set of constraints. These issues will be applied in the context of the PLUG project which aims at creating an ubiquitous affective game that will take place in a museum.
\end{abstract}

Keywords: Affective computing, biofeedback, physiology, emotion.

\section{Introduction}

During the last fifteen years, the theoretical background on human emotion mechanisms has dramatically evolved. Whereas it was formerly considered as a non scientific matter, it is now obvious that emotions play a significant role in decision making.

In 1887, James and Lange proposed a theory of emotions suggesting that emotion is largely due to the experience of bodily changes.

In 1929, Cannon and Bard suggest another theory arguing that emotion triggers or stimulates behaviors, as opposed to James-Lange theory.

In Lazarus' theory (appraisal theory - 1991), even if some emotions can be triggered unconsciously, emotions arise from the conscious cognitive evaluation of an event. Scherer further developed the appraisal theory of emotions.

Damasio studied patients having decision-making and emotional disorders due to localized brain damage causing both a degradation in the ability to feel and express emotions and decision-making troubles. His observations led him to the hypothesis that emotions play a biasing role in decision making, probably acting a bit like a heuristic: preventing an infinite exploration of rational possibilities and evaluating outcomes a priori.

Based on this idea that emotions are needed for rational behavior, Picard defined a field of study for computer science focused on the implication of emotion in Human Computer Interactions (HCI) called Affective Computing.

\section{Previous Work}

\subsection{Affective Computing}

Wastell and Newman showed that physiological measures have a valuable role to play in system validation. 
Huber et al. and Ang et al. used prosodic speech analysis to detect anger in utterances respectively with acted data and naturally occurring data.

D'mello et al. built an intelligent tutoring system using affect-sensing technologies (facial expressions, posture information, conversational cues).

Meehan et al. studied presence in stressful virtual environments using physiological measurement (Heart Rate (HR), Galvanic Skin Resistance (GSR) and Skin Temperature).

\subsection{Affective Gaming}

Iida et al. developed a metric of entertainment for board games only based on the characteristics of the game using variants of chess game.

Gerasimov used physiological measurement as a gameplay mechanism towards personal health monitoring.

Syked and Brown correlated gamepad interactions during play experience with the difficulty of the game. They showed that the gamepad provides physiological information on the user, though further study is needed.

Mandryk et al. used fuzzy logic to extract arousal/valence information from physiological data successfully correlated with subjective emotional evaluations of subjects (e.g. GSR with fun, Electromyogram (EMG) with challenge).

Beaume et al. used game states as a measure of flow in Pac-man game to evaluate fun and difficulty in the game.

\section{Researches}

We expect that the comparative study of physiological signals acquired in conditions that allow comparison, from normalized stimuli, would permit to determine the set of sensors that best fits specific constraints. From that, we intend to extract a smaller set of physiological measures that are compatible with mobility in terms of intrusivity, encumbrance and dataflow, and focus on noise resistance for these specific biofeedbacks.

An experimental framework for the study of the effect of emotions on physiological measures would also enable the comparison with other measures such as measures on the standard interaction which could complement more intrusive measures.

The evaluation of user reactions to software events opens the possibility of developing new strategies of adaptation and optimization towards user-friendliness. It also allows to consider using physiological data for design validation for software development which is a crucial issue generally limited to subjective report today. 